\title{
PAPER \\ MIMO E-SDM Transmission Performance in an Actual Indoor Environment
}

\author{
Hiroshi NISHIMOTO $^{\dagger a)}$, Student Member, Yasutaka OGAWA ${ }^{\dagger \mathrm{b})}$, Toshihiko NISHIMURA $^{\dagger c)}$, \\ and Takeo OHGANE ${ }^{\dagger \mathrm{d})}$, Members
}

\begin{abstract}
SUMMARY MIMO systems using a space division multiplexing (SDM) technique in which each transmit antenna sends an independent signal substream have been studied as one of the successful applications to increase data rates in wireless communications. The throughput of a MIMO channel can be maximized by using an eigenbeam-SDM (E-SDM) technique, and this paper investigates the practical performance of $2 \times 2$ and $4 \times 4$ MIMO E-SDM based on indoor measurements. The channel capacity and bit error rate obtained in various uniform linear array configurations are evaluated and are compared with the corresponding values for conventional SDM. Analysis results show that the bit error rate performance of E-SDM is better than that of SDM and that E-SDM gives better performance in line-of-sight (LOS) conditions than in non-LOS ones. They also show that the performance of E-SDM in LOS conditions depends very much on the array configuration.

key words: $M I M O$, eigenbeam space division multiplexing, array element pattern, eigenvalue distribution, channel capacity, bit error rate
\end{abstract}

\section{Introduction}

The multiple-input multiple-output (MIMO) system in which multiple antennas are placed at both the transmitting and receiving ends has recently been shown to be effective in providing high-capacity wireless communications [1]-[3], and its propagation characteristics have been measured by many groups of investigators [4]-[11]. Space division multiplexing (SDM), in which data rates are increased in proportion to the number of transmit antennas by having each send an independent signal substream, can provide high-speed data transmission even when the MIMO channel state information (CSI) is unknown at the transmitting end [12]-[14]. We have already made MIMO channel measurements evaluating the performance of SDM in actual environments [15], [16].

When the MIMO CSI is available at the transmitting end, on the other hand, eigenbeam-SDM (E-SDM) can be used [17]-[21]. This transmission technique enables us to transmit spatially orthogonal substreams when eigenvectors obtained by singular value decomposition of the MIMO channel matrix are used as transmit weight vectors, and it

\footnotetext{
Manuscript received August 4, 2006.

Manuscript revised January 6, 2007.

†The authors are with the Graduate School of Information Science and Technology, Hokkaido University, Sapporo-shi, 0600814 Japan.

a)E-mail: hn@ist.hokudai.ac.jp

b) E-mail: ogawa@ist.hokudai.ac.jp

c) E-mail: nishim@ist.hokudai.ac.jp

d)E-mail: ohgane@ist.hokudai.ac.jp

DOI: $10.1093 /$ ietcom/e90-b.6.1474
}

maximizes the throughput of the MIMO channel by allocating resources to each substream optimally. These advantages of E-SDM will make MIMO systems effective for increasing the data transfer speeds in future wireless communications.

To assess the feasibility of E-SDM in actual propagation environments, we have measured MIMO channel properties in indoor line-of-sight (LOS) and non-LOS (NLOS) environments. Some measurement-based MIMO researches have been done with respect to virtual channels, especially Kronecker reconstructed channels [5], [6], [9]. When the number of measured channel samples is insufficient for a statistical analysis, such a channel production scheme is effective because it can infinitely generate MIMO channels based on the measured data. Statistical characteristics of generated channels, however, may differ from those in the actual measurement environment [22]. Furthermore, the Kronecker reconstruction is generally used to produce virtual NLOS channels so that LOS channels, which are evaluated in our study, are out of the consideration.

We think that the MIMO characteristics in a target environment should be investigated using the measured channels themselves as much as possible. Therefore we have obtained enough actual channel samples for statistical analyses by collecting data in both the space and frequency domains [6]. This measurement scheme enables the MIMO characteristics in real environments to be analyzed more accurately. Using the measured channels including the effect of mutual coupling between antennas, we evaluated $2 \times 2$ and $4 \times 4$ MIMO E-SDM performance in an indoor environment.

The following parts of this paper are organized as follows. Section 2 defines the model of the MIMO channel and introduces the basic concepts of SDM and E-SDM. Section 3 outlines our measurement campaign and describes some examples of measured data and characteristics of measurement environments. Section 4 reports the channel capacity and average bit error rate (BER) performance obtained by using the measured data. It also shows the array element patterns including the effect of mutual coupling and the cumulative distribution functions (CDFs) of eigenvalues so the relations between these properties and the obtained performance can be evaluated. In Sect. 5 we draw conclusions from these results. 


\section{Concepts of SDM and E-SDM}

\subsection{MIMO Channel Model}

We assume that the transmission bandwidth is so narrow that the fading is flat. When the MIMO system is assumed to have $N_{T}$ transmit (TX) antennas and $N_{R}$ receive $(\mathrm{RX})$ antennas, an $N_{R} \times N_{T}$ MIMO channel matrix $\boldsymbol{H}$ between the TX and RX ends is expressed as

$$
\boldsymbol{H}=\left[\begin{array}{cccc}
h_{11} & h_{12} & \cdots & h_{1 N_{T}} \\
h_{21} & h_{22} & \cdots & h_{2 N_{T}} \\
\vdots & \vdots & h_{i j} & \vdots \\
h_{N_{R} 1} & h_{N_{R} 2} & \cdots & h_{N_{R} N_{T}}
\end{array}\right],
$$

where $h_{i j}$, which is an element of the $i$ th row and the $j$ th column in the matrix $\boldsymbol{H}$, denotes a channel from the $j$ th TX antenna to the $i$ th $\mathrm{RX}$ antenna.

\section{$2.2 \mathrm{SDM}$}

When the transmitter does not know the MIMO CSI, highspeed communications can be obtained by using SDM, in which independent signal substreams are transmitted from the TX antennas with equal power as shown in Fig. 1(a). In SDM transmission, an $N_{R}$-dimensional received signal vector $\boldsymbol{r}_{\mathrm{SDM}}(t)$ can be represented by

$\boldsymbol{r}_{\mathrm{SDM}}(t)=\boldsymbol{H} \boldsymbol{s}(t)+\boldsymbol{n}(t)$,

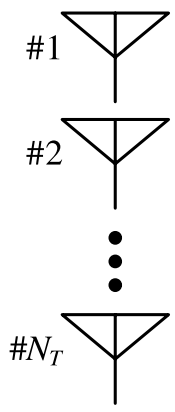

TX antennas
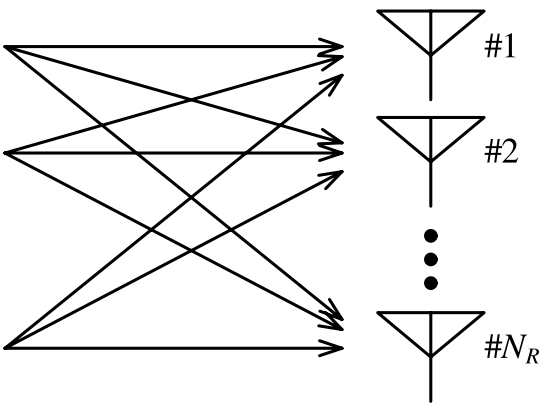

(a) SDM.

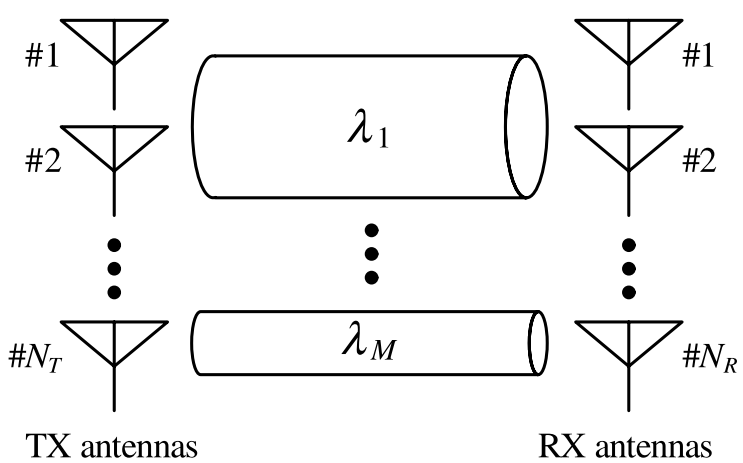

(b) E-SDM. where $s(t)$ is an $N_{T}$-dimensional transmitted signal vector consisting of signals from TX antennas and $\boldsymbol{n}(t)$ is an $N_{R^{-}}$ dimensional additive white Gaussian noise vector. While SDM can increase data rates in proportion to the number of TX antennas, the receiver needs to suppress intersubstream interference.

\subsection{E-SDM}

If the MIMO CSI is available at the transmitter, the channel space between the TX and RX antennas can be orthogonalized by using eigenbeams. The eigenvalue decomposition of an $N_{T} \times N_{T}$ non-negative Hermitian matrix $\boldsymbol{H}^{H} \boldsymbol{H}$ can be expressed as

$$
\boldsymbol{H}^{H} \boldsymbol{H}=\boldsymbol{U} \boldsymbol{\Lambda} \boldsymbol{U}^{H},
$$

where

$$
\begin{aligned}
\boldsymbol{U} & =\left[\boldsymbol{e}_{1} \boldsymbol{e}_{2} \cdots \boldsymbol{e}_{M}\right], \\
\boldsymbol{\Lambda} & =\operatorname{diag}\left(\lambda_{1}, \lambda_{2}, \ldots, \lambda_{M}\right) \quad\left(\lambda_{1} \geq \cdots \geq \lambda_{M}\right) .
\end{aligned}
$$

Here, $M\left(M \leq \min \left(N_{T}, N_{R}\right)\right)$ indicates the rank of $\boldsymbol{H}$, $\operatorname{diag}(\cdot)$ denotes a diagonal matrix, and $\boldsymbol{\Lambda}$ is defined as an $M \times M$ diagonal matrix composed of positive eigenvalues $\lambda_{1}, \ldots, \lambda_{M}$ of $\boldsymbol{H}^{H} \boldsymbol{H}$. When eigenvectors $\boldsymbol{e}_{1}, \ldots, \boldsymbol{e}_{M}$ respectively corresponding to eigenvalues $\lambda_{1}, \ldots, \lambda_{M}$ are multiplied as TX weights, we can form an orthogonal multibeam space between the TX and RX antennas as shown in Fig. 1(b). This is the E-SDM technique, and when it is used the received signal vector is given by

$$
\boldsymbol{r}_{\mathrm{E}-\mathrm{SDM}}(t)=\boldsymbol{H} \boldsymbol{U} \boldsymbol{s}(t)+\boldsymbol{n}(t) .
$$

Unlike the $s(t)$ in (2), $s(t)$ in this equation is an $M$ dimensional transmitted signal vector consisting of signals sent through eigenchannels. In the ideal E-SDM in which the TX weight vectors completely match an instantaneous MIMO channel response, spatially orthogonal substreams cause no intersubstream interference at the RX end, which is an issue in the conventional SDM. Under such a condition, it is easy to demultiplex received signals by using maximal ratio combining (MRC) processing, which maximizes the SNR at the RX end by multiplying the received signal vector $\boldsymbol{r}_{\mathrm{E}-\mathrm{SDM}}(t)$ by $(\boldsymbol{H} \boldsymbol{U})^{H}$ [23]. Moreover, we can maximize the throughput of the MIMO system by optimizing bit (modulation) and power allocation to each substream.

We measured MIMO transfer matrices $\boldsymbol{H}$ themselves in actual indoor propagation environments as described in the next section. Using the measured channels, we evaluated the performance of E-SDM and compared it with the performance of the conventional SDM as described in Sect. 4.

\section{MIMO Channel Measurement Setup}

The measurement campaign was carried out in a conference room in a building of the Graduate School of Information Science and Technology at Hokkaido University.

Fig. 1 Concepts of SDM and E-SDM transmission. 


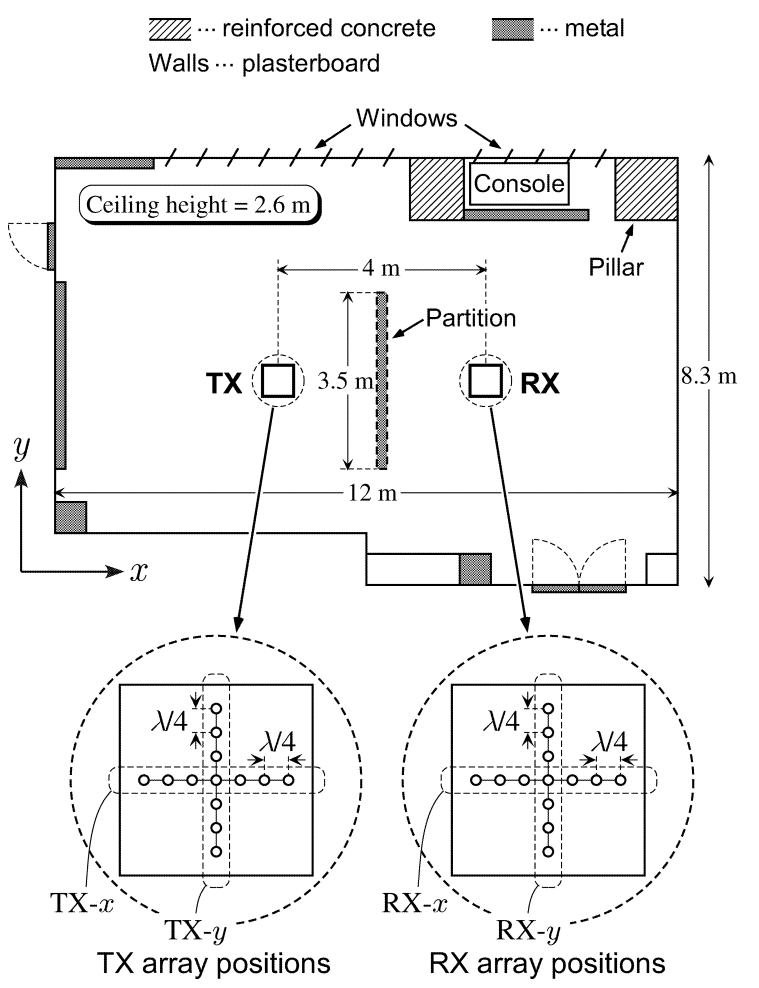

Fig. 2 Measurement environment (top view).

The walls of the room consisted of plasterboard around reinforced concrete pillars and metal doors (Fig. 2). The walls, floor and ceiling scatter a transmitted signal and cause fading. In it we set up TX and RX tables and a vector network analyzer (VNA) to measure the channel responses. The measurement band was from $5.15 \mathrm{GHz}$ to $5.4 \mathrm{GHz}$ (bandwidth $=250 \mathrm{MHz}$ ), and it was swept with a $156.25 \mathrm{kHz}$ interval (1,601 frequency sample points). Each channel was averaged over 10 snapshots in order to reduce thermal noise included in tentative measurements. In the LOS condition there was no obstructing object between the TX and RX tables, and in the NLOS condition there was a metal partition between them. Channel data were obtained while no one was in the room, to ensure statistical stationarity of propagation.

The $x$ - and $y$-axes were defined as shown in the dashed circles in Fig. 2. The MIMO measurement campaign used uniform linear arrays (ULAs). The orientation with the TX and $\mathrm{RX}$ antennas aligned along the $x$-axis is denoted as the TX- $x /$ RX- $x$ orientation (Fig. 3(a)), and the orientation with the TX and RX antennas aligned along the $y$-axis is denoted as the $\mathrm{TX}-y / \mathrm{RX}-y$ orientation (Fig. 3(b)). To obtain spatially different fading channels, there were seven positions on the TX and RX tables for the antenna array mount along the $x$ - and $y$-axes separated with an interval of $\lambda / 4(1.5 \mathrm{~cm})$, as shown in the dashed circles in Fig. 2, where $\lambda$ denotes the wavelength at $5 \mathrm{GHz}(6 \mathrm{~cm})$. As also shown in the dashed circles in Fig. 2, the antenna array orientation corresponded to the array position direction. The positions of the TX and the RX arrays for the TX- $x / \mathrm{RX}-x$ orientation, for exam-
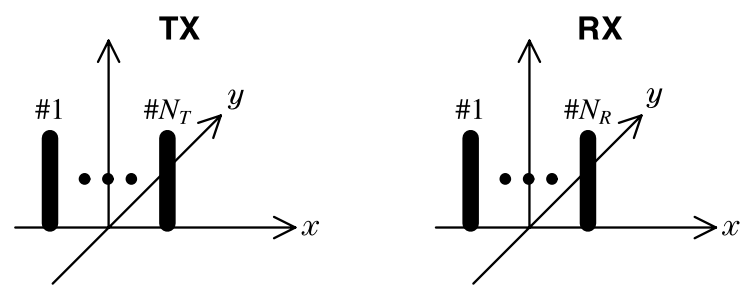

(a) $\mathrm{TX}-x / \mathrm{RX}-x$
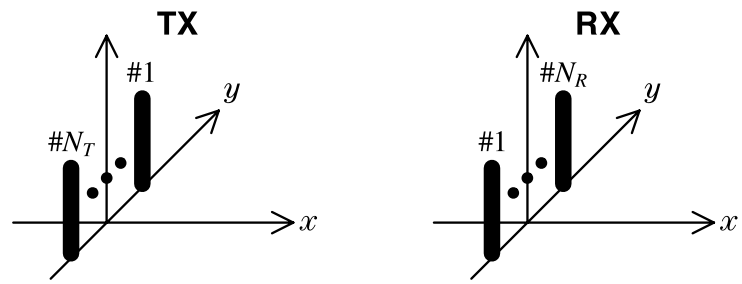

(b) TX-y/RX-y.

Fig. 3 Antenna array orientations.

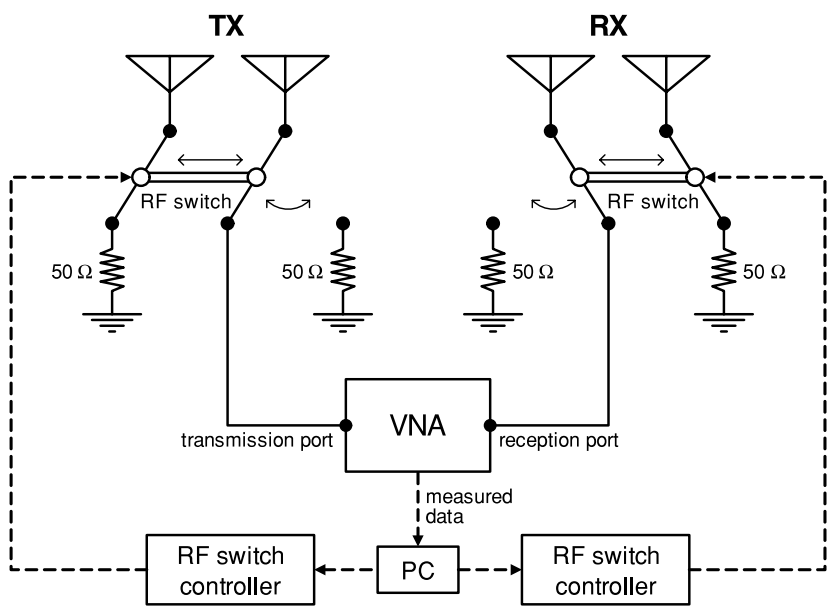

Fig. 4 MIMO channel measurement system.

ple, were changed in the $x$ direction. The array's antenna spacing (AS) had three values: $0.50 \lambda, 0.75 \lambda$, and $1.00 \lambda$. By changing the TX and RX array positions, we obtained $7 \times 7=49$ spatially different data. Because we had 1,601 frequency-domain data, a total of $49 \times 1,601=78,449$ different MIMO channel matrix data were obtained for each array orientation, antenna spacing, and LOS/NLOS condition. That is, strictly speaking, we measured $\hat{\boldsymbol{H}}(k, f)$ for the space-domain index $k=1, \ldots, 49$ and the frequencydomain index $f=1, \ldots, 1,601$. In our study we treated the 78,449 channel matrices independently as flat fading channels based on (1). All of the MIMO characteristics presented in the next section were derived from statistical processing of all the 78,449 MIMO channel data.

We used collinear antennas AT-CL010 (TSS JAPAN Co., Ltd.) designed to have omnidirectional characteristics on the horizontal $(x-y)$ plane. The nominal gain on the horizontal plane was $4 \mathrm{dBi}$. Figure 4 shows an example of a $2 \times 2$ MIMO measurement system, where the basic idea is the same as for the $4 \times 4$ MIMO system. We set and mea- 


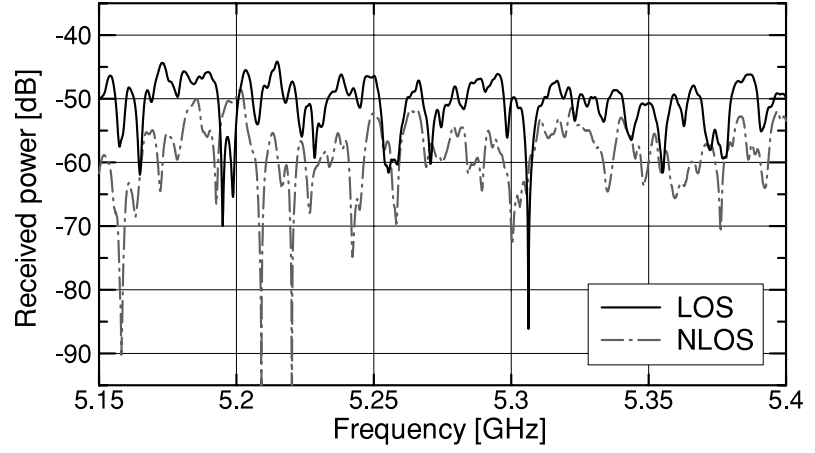

(a) Frequency domain.

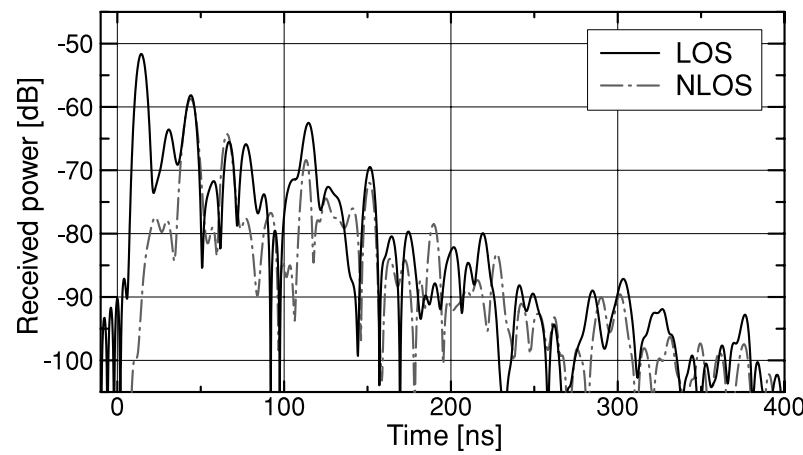

(b) Time domain.

Fig. 5 Examples of measurement data (SISO case).

sured a target channel element in the MIMO matrix by using RF switches at the TX and RX sides to select the TX antenna and the RX antenna. We normalized the measured channel responses to calibration data obtained when the cables to the antenna ports from the RF switches were connected directly. The calibrated data therefore did not have the frequency characteristics of the cables and switches. The unselected antennas were automatically connected to $50 \Omega$ dummy loads.

Figure 5 shows example measurements. These data were obtained at the central positions on the TX and RX tables when TX and RX ends each used a single antenna (single-input single-output (SISO) case), so the antennas did not have mutual coupling. Figure 5(a) shows measured frequency-domain data. It is clear that the received power for the LOS condition was generally larger than that for the NLOS one because of the direct wave. The impulse responses in the time domain shown in Fig. 5(b) are the result of performing an IFFT on the frequency-domain data in Fig. 5(a). The many peaks for the LOS and NLOS conditions indicate that the measurement environments had many scattered waves. The maximum peak was around $14 \mathrm{~ns}$ for the LOS condition. This is considered to be from the direct wave because the 4-m distance between the TX and RX ends gives a propagation time of $4 /\left(3.0 \times 10^{8}\right)=13.3 \mathrm{~ns}$ and because the peak was not present under the NLOS condition.

Figure 6 shows the CDFs of the wave amplitudes. The data were obtained with the SISO technique. The received amplitudes at the $50 \%$ level were about $7.6 \mathrm{~dB}$ greater under

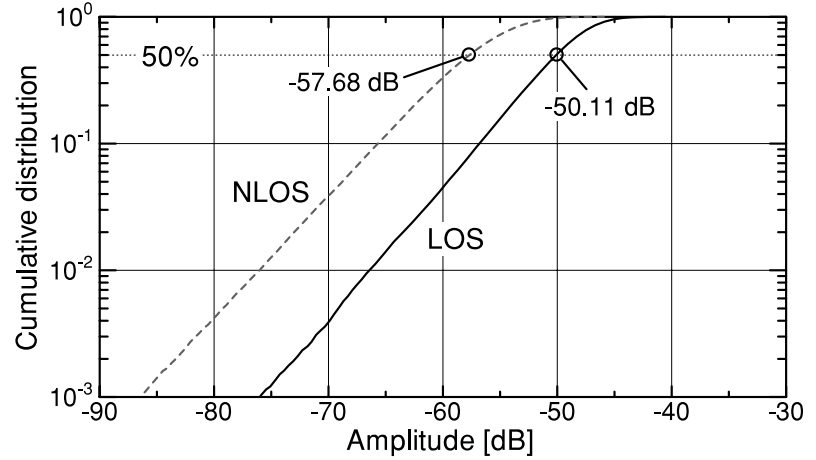

Fig. 6 CDFs of amplitudes (SISO case).

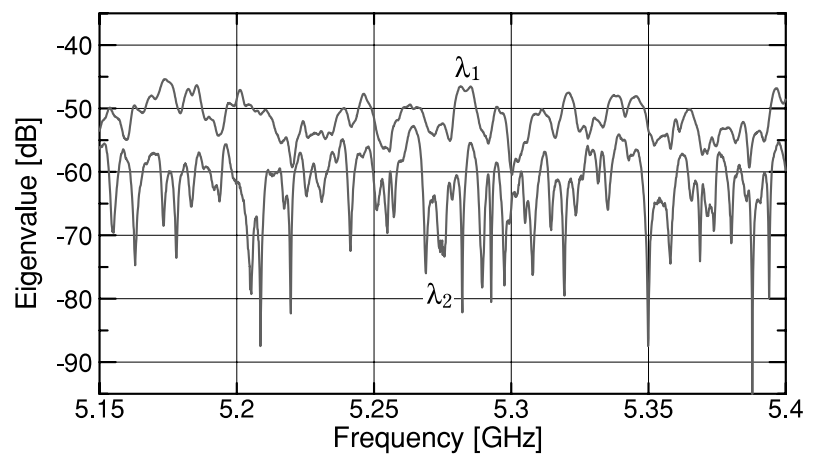

(a) NLOS.

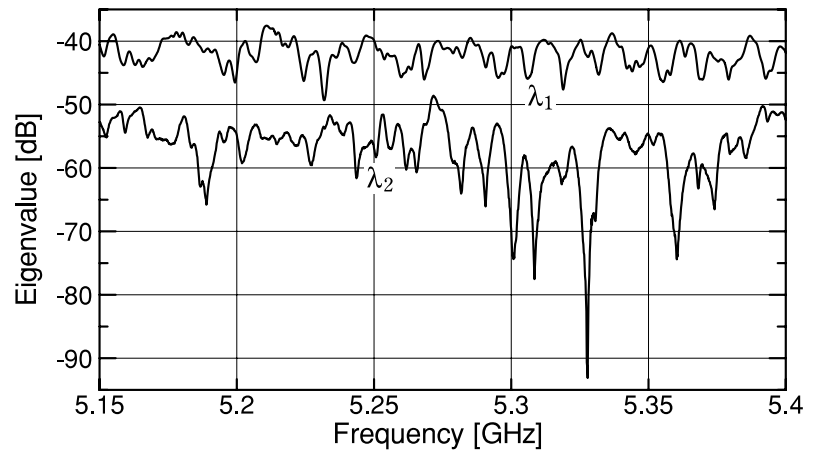

(b) LOS.

Fig. 7 Examples of eigenvalues for the $2 \times 2 \mathrm{MIMO}$ case $(\mathrm{AS}=0.50 \lambda$, $\mathrm{TX}-y / \mathrm{RX}-y$ orientation).

the LOS condition than they were under the NLOS condition. The $K$-factor in the LOS environment was verified to be $1.66 \mathrm{~dB}$. Therefore, the scattered wave power was comparable to the direct wave power.

Figure 7 shows examples of eigenvalues for the $2 \times 2 \mathrm{MIMO}$ case with AS $=0.50 \lambda$ and the TX- $y / \mathrm{RX}-y$ orientation. These data were obtained at the same position that those in the abovementioned SISO case were obtained. In contrast to the antennas in the SISO case, the antennas in this case had the effect of mutual coupling. Figure 7(a) shows eigenvalues in the NLOS condition, and Fig. 7(b) shows those in the LOS one. There are two eigenvalues $\lambda_{1}$ and $\lambda_{2}$ because of a $2 \times 2$ MIMO system. Under both of the conditions the change of $\lambda_{1}$ is smaller than that of $\lambda_{2}$ (as will be 
seen also from Figs. 10 and 11 in Sect. 4.2). We can expect that spatial diversity appears significantly for the eigenchannel corresponding to the maximum eigenvalue. We can also see that the LOS condition gives a larger $\lambda_{1}$ than the NLOS one does. As in the SISO case, the direct wave in the LOS case seems to have increased the magnitude of $\lambda_{1}$.

The MIMO channel matrix $\boldsymbol{H}$ used in the next section is given by the following normalization of the measured $\hat{\boldsymbol{H}}$ :

$$
\boldsymbol{H}=\frac{\hat{\boldsymbol{H}}}{\sqrt{E\left[\mid h_{\left.\mathrm{AEC}\right|^{2}}\right]}},
$$

where $E[\cdot]$ denotes a mean value averaged over frequency and space samples. $E\left[\left|h_{\mathrm{AEC}}\right|^{2}\right]$ is the mean received power obtained under the SISO and LOS conditions in an anechoic chamber in which the centers of the TX and RX tables are $4 \mathrm{~m}$ apart. We can say that the channel response is normalized to the direct wave amplitude.

\section{Analysis of Measured Data}

\subsection{Array Element Patterns}

When multiple antennas are closely arrayed, they have mutual coupling and their antenna patterns change [24]. Since a MIMO system has antenna arrays at both ends, we cannot ignore the effect of the changed pattern on the MIMO performance. So before presenting the measured characteristics of the MIMO channels, we examine the antenna patterns for each antenna array.

The solid curves in Figs. 8 and 9 show the patterns at the frequency of $5.2 \mathrm{GHz}$ for each two-element array and four-element ULA, respectively. The number under each pattern corresponds to the one in Fig. 3. These azimuth patterns for multiple antennas were obtained when all the antennas except the measured one were connected to $50 \Omega$ dummy loads. Therefore they include the mutual coupling effect. In each part of these figures, the pattern of a single isolated antenna is shown for comparison by a dashed curve. We see that the single antenna has an almost omnidirectional pattern when it does not have the mutual coupling effect. The patterns in the multiple-antenna case, however, are significantly different from those in the omnidirectional case (The patterns for AS $=0.50 \lambda$ and $1.00 \lambda$ in the twoelement arrays are in good agreement with the simulation results in Ref. [24]). The patterns tend to become similar to the omnidirectional one as the AS becomes larger. We can also see that patterns of four-element ULAs tend to change more than those of two-element arrays. This seems to be due to the effect of mutual coupling between antennas increasing with the number of antennas.

In the TX- $x / \mathrm{RX}-x$ orientation, the $\mathrm{RX}$ end is located in the $0^{\circ}$ direction with respect to the TX end, and the TX end is located in the $180^{\circ}$ direction with respect to the $\mathrm{RX}$ end. Thus, in the LOS condition, the direct wave departs from the TX end in the $0^{\circ}$ direction and arrives at the RX end in the $180^{\circ}$ direction. In the TX-y/RX- $y$ orientation, on the other
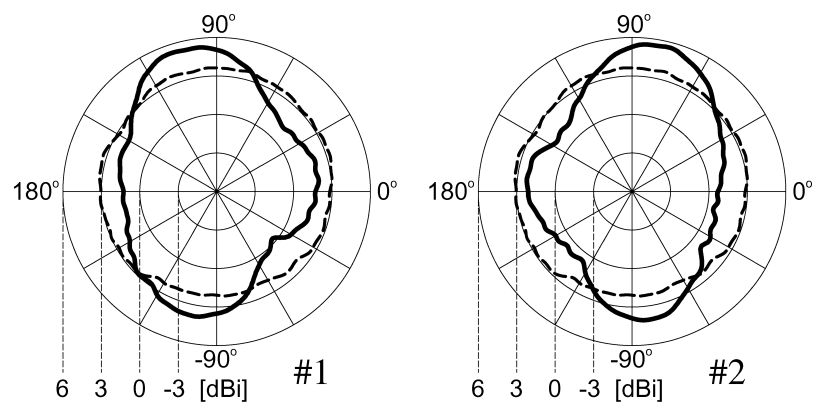

(a) $\mathrm{AS}=0.50 \lambda$
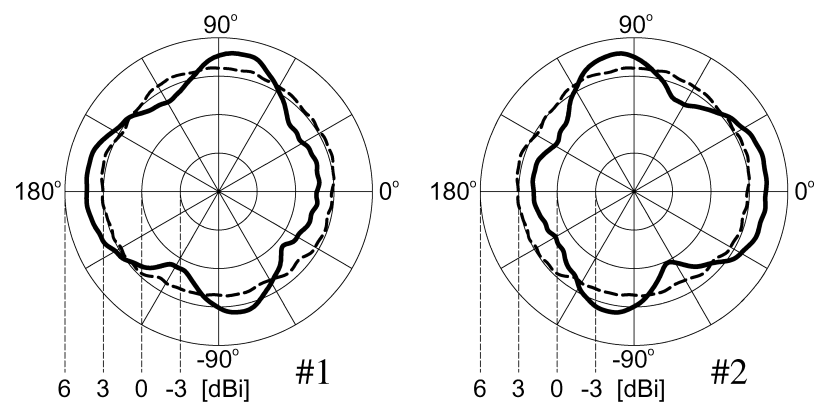

(b) $\mathrm{AS}=0.75 \lambda$
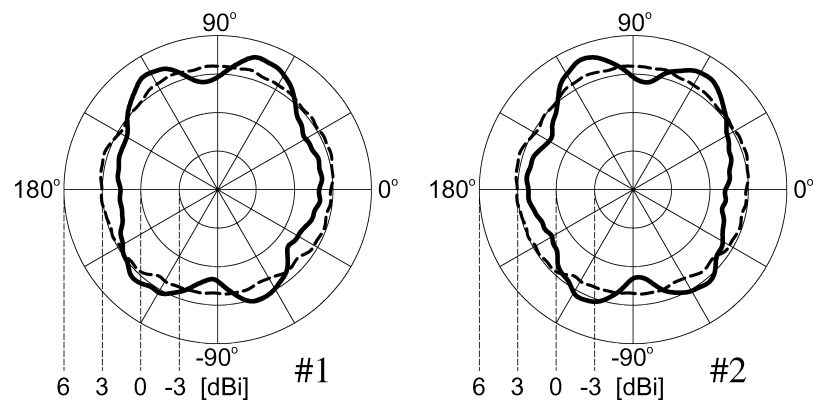

(c) $\mathrm{AS}=1.00 \lambda$

Fig. 8 Antenna patterns for a single antenna (dashed curves) and for each two-element array with mutual coupling (solid curves).

hand, the RX end is located in the $90^{\circ}$ direction with respect to the TX end, and the TX end is also located in the $90^{\circ}$ direction with respect to the RX end. Thus the direct wave departs from the TX end and arrives at the RX end in the $90^{\circ}$ direction. The gain in the $0^{\circ}$ and $180^{\circ}$ directions tends to be small. On the other hand, the gain in the $90^{\circ}$ direction is higher than it is in the single-antenna case, especially when $\mathrm{AS}=0.50 \lambda$ and $0.75 \lambda$.

As will be shown later, especially in the LOS condition, eigenvalue distributions and MIMO system performance depend on the array configuration. The antenna gain directionality mentioned above seems to affect the LOS component included in a MIMO channel. Because the relation between the LOS component and antenna gain directionality is necessary to explain the following behavior of MIMO performance, we call it "LOS gain variation" to simplify the following discussions.

\subsection{CDFs of Eigenvalues}

The number of positive eigenvalues of an $N_{T} \times N_{T}$ Hermitian 

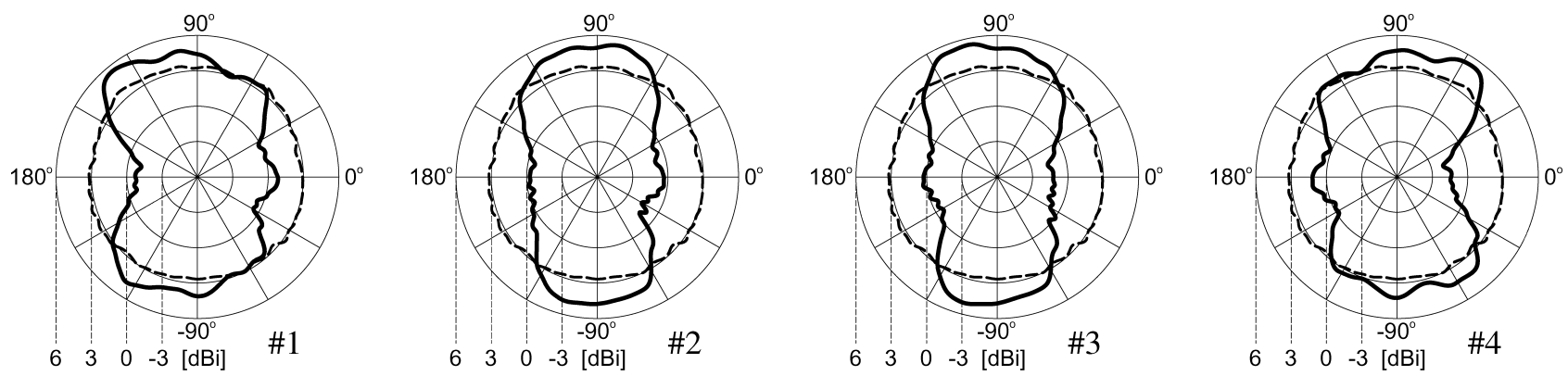

(a) $\mathrm{AS}=0.50 \lambda$
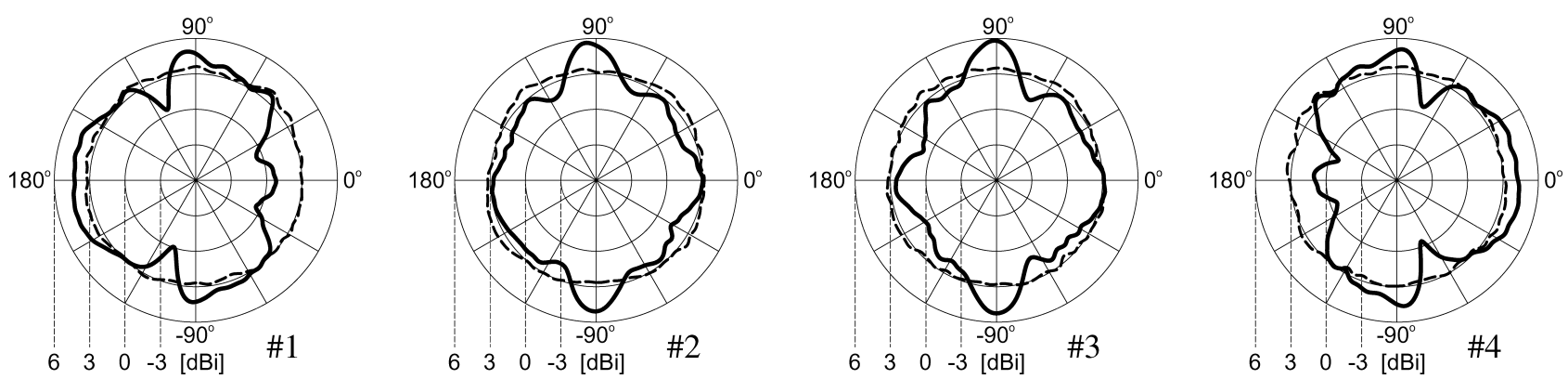

(b) $\mathrm{AS}=0.75 \lambda$
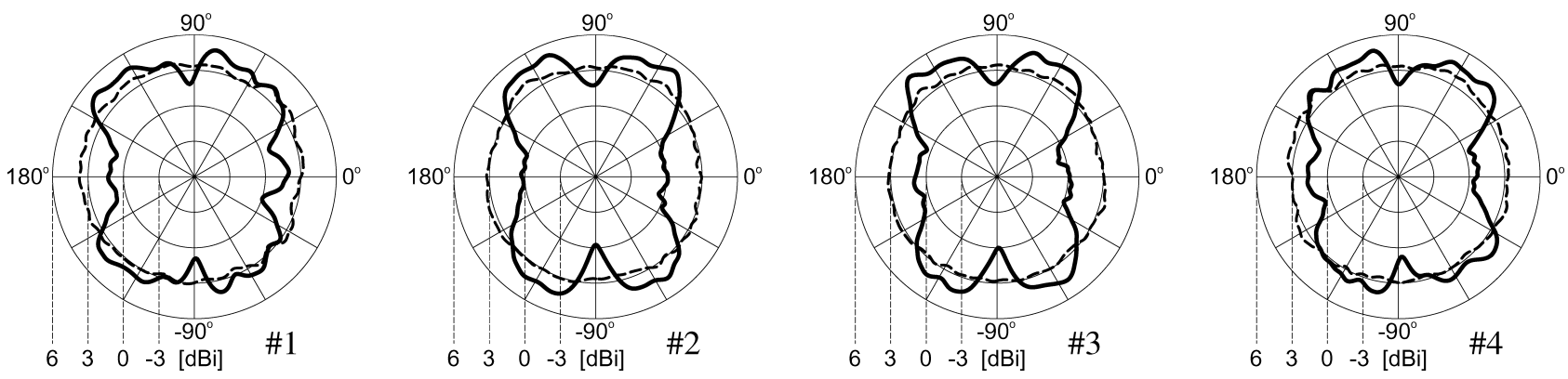

(c) $\mathrm{AS}=1.00 \lambda$

Fig. 9 Antenna patterns for a single antenna (dashed curves) and for each four-element ULA with mutual coupling (solid curves).

matrix $\boldsymbol{H}^{H} \boldsymbol{H}$ corresponds to the number of available eigenchannels between the TX and RX ends, and the magnitude of the eigenvalue is proportional to the SNR of the corresponding channel. This means that data transfer rates can be increased with the number of large eigenvalues. The number and magnitude of positive eigenvalues therefore determine the transmission performance in E-SDM. On the other hand, the channel capacity in SDM is also given by eigenvalues as will be described in Sect. 4.3. It has also been reported that the average BER performance of SDM tends to be determined by the minimum eigenvalue $\lambda_{N_{T}}$ [15], [16], [26]. Eigenvalues are considered to have large influence on the performance in not only E-SDM but also SDM. We therefore examined the CDFs of eigenvalues $\lambda_{1}, \ldots, \lambda_{N_{T}}$ in each MIMO configuration.

The CDFs of the eigenvalues for the measured $2 \times 2$ and $4 \times 4$ MIMO channels are shown in Figs. 10 and 11, respectively. Figures 10 (a) and (b) are for the TX- $x / \mathrm{RX}-x$ and TX- $y / \mathrm{RX}-y$ orientations. In Fig. 11, parts (a) and (b) are for the TX- $x / \mathrm{RX}-x$ orientation and parts (c) and (d) are for the TX- $y / \mathrm{RX}-y$ orientation. In addition, parts (a) and (c) are for the LOS condition and parts (b) and (d) are for the NLOS condition. We can see from these figures that the eigenvalues in the LOS condition are generally larger than those in the NLOS one. In particular, the maximum eigenvalues $\lambda_{1}$ in the LOS condition become significantly large. Since the direct wave in the LOS condition increases the channel power, its effect seems to largely appear in $\lambda_{1}$.

Comparing the CDFs in each condition, we can see that although the NLOS condition gives similar distributions in all the cases, the CDFs in the LOS condition, particularly those of the maximum eigenvalues $\lambda_{1}$, change depending on the array configuration. We can explain this by the relation between the direct wave and antenna patterns shown in Figs. 8 and 9, i.e., the LOS gain variation stated in the previous subsection. The effect of LOS gain variation is especially evident in the magnitude of the maximum eigenvalue $\lambda_{1}$, as mentioned before. That is, when the antennas have higher gain in the LOS direction, $\lambda_{1}$ tends to be larger. In contrast, when the antennas have lower gain in the LOS di- 


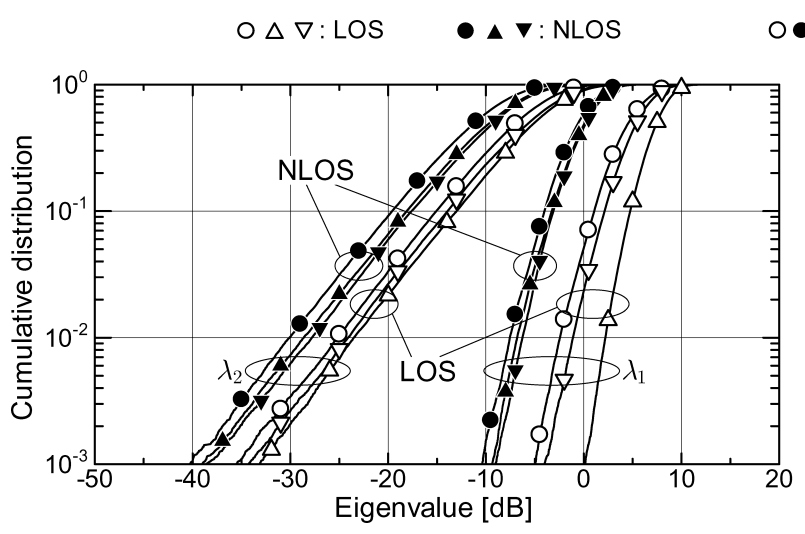

(a) TX- $x / \mathrm{RX}-x$.
: AS $=0.50 \lambda \quad \Delta \Delta: A S=0.75 \lambda \quad \nabla \nabla: A S=1.00 \lambda$

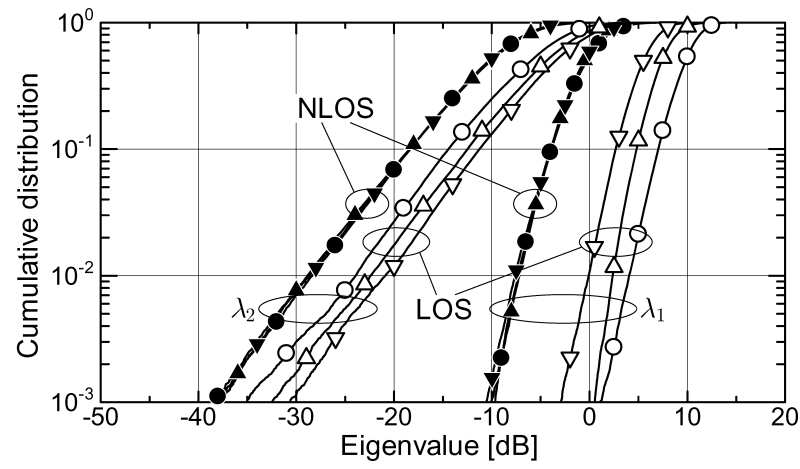

(b) TX-y/RX- $y$.

Fig. 10 CDFs of eigenvalues for the measured $2 \times 2$ MIMO channels.

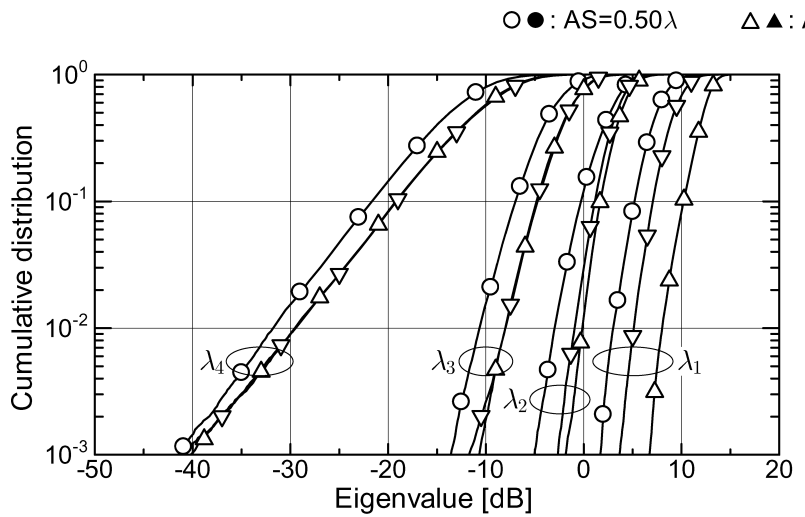

(a) TX- $x / \mathrm{RX}-x$, LOS.

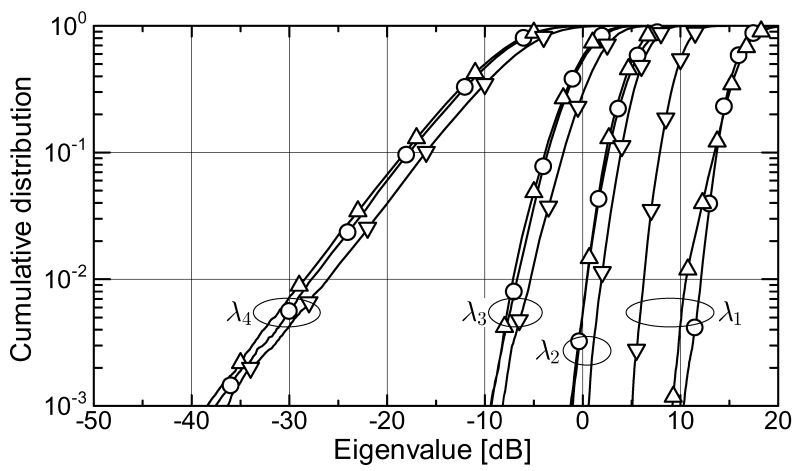

(c) TX- $y /$ RX- $y$, LOS.

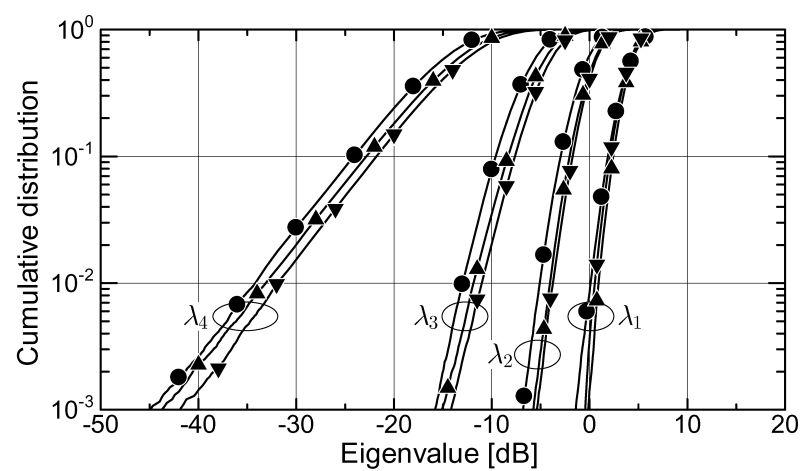

(b) TX- $x /$ RX- $x$, NLOS.

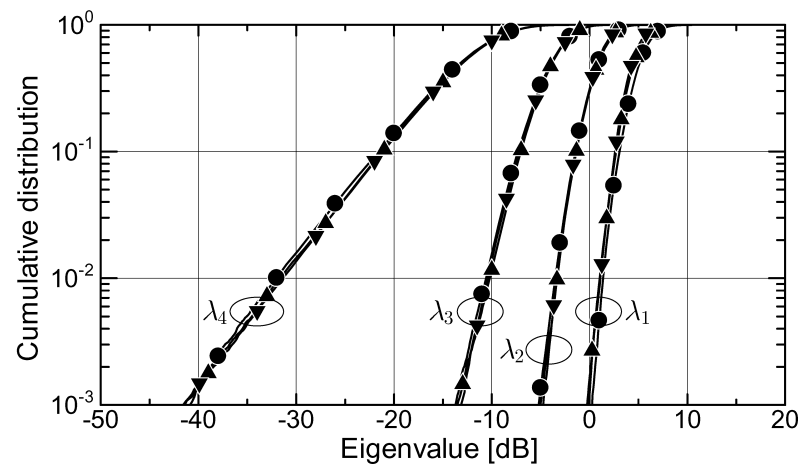

(d) TX-y/RX- $y$, NLOS.

Fig. 11 CDFs of eigenvalues for the measured $4 \times 4$ MIMO channels.

rection, $\lambda_{1}$ tends to be lower. For example, looking at the case of the TX- $y / \mathrm{RX}-y$ array orientation in the LOS condition, we can see that the arrays of $\mathrm{AS}=0.50 \lambda$ and $0.75 \lambda$ have large $\lambda_{1}$ for both of the $2 \times 2$ and $4 \times 4$ MIMO systems. In the case of $\mathrm{AS}=1.00 \lambda$, on the other hand, the CDF of $\lambda_{1}$ is lower than in the above two cases. As will be presented later, such variations of $\lambda_{1}$ in the LOS condition appear to yield various MIMO performances, particularly E-SDM performances.

We can notice that the CDF of the minimum eigenvalue $\lambda_{N_{T}}$ is in a clearly lower magnitude region and is far apart from those of the other eigenvalues. Also, the gradients of the minimum eigenvalue CDFs show almost an order of magnitude increase with an eigenvalue increment of $10 \mathrm{~dB}$. In other words, an eigenchannel with the minimum eigenvalue does not have spatial diversity effect whereas the other eigenchannels have. We confirmed that the same phenomena are observed for the i.i.d. Gaussian MIMO channels generated in computer simulations. This appears to cause a difference in substream utilization in E-SDM between channel capacity and BER as will be discussed in Sect. 4.4. 


\subsection{CDFs of Channel Capacities}

The channel capacity has been used extensively for evaluating the MIMO channels [4]-[11]. It is the maximum amount of information that could be transferred if we used an ideal communication method (coding and modulation). When the MIMO CSI is known only at the RX side, the SDM channel capacity $C_{\mathrm{SDM}}$ of the measured MIMO channel is given by

$$
\begin{aligned}
C_{\mathrm{SDM}} & =\log _{2}\left\{\operatorname{det}\left(\boldsymbol{I}+\frac{P_{\text {total }}}{P_{\mathrm{AEC}}} \frac{1}{N_{T}} \boldsymbol{H}^{H} \boldsymbol{H}\right)\right\} \\
& =\sum_{i=1}^{N_{T}} \log _{2}\left(1+\frac{P_{\text {total }}}{P_{\mathrm{AEC}}} \frac{1}{N_{T}} \lambda_{i}\right) .
\end{aligned}
$$

Here $\operatorname{det}(\cdot)$ denotes a determinant, $P_{\text {total }}$ is the total TX power, and $P_{\mathrm{AEC}}$ is the TX power when the previously mentioned SISO measurement in the anechoic chamber gives an average received $E_{s} / N_{0}$ of $0 \mathrm{~dB}$. Note that $P_{\text {total }} / P_{\mathrm{AEC}}$ here represents the normalized total TX power, which is used hereafter to evaluate the channel capacities and bit error rates (BERs) so we can compare performances under the same total TX power condition.

When the MIMO CSI is available at both the TX and RX sides, we can employ E-SDM transmission that uses orthogonal eigenchannels. In this case, optimizing power allocation to each channel gives the maximum channel capacity in the MIMO channel. The capacity is given by

$$
C_{\mathrm{E}-\mathrm{SDM}}=\sum_{m=1}^{M} \log _{2}\left(1+\frac{P_{\text {total }}}{P_{\mathrm{AEC}}} p_{m} \lambda_{m}\right) .
$$

Here the power coefficient $p_{m}(m=1, \ldots, M)$ allocated to the $m$ th eigenchannel is optimally determined on the basis of the water-filling (WF) theorem [3], [25] represented as

$$
p_{m}=\max \left(\eta-\frac{P_{\mathrm{AEC}}}{P_{\text {total }}} \frac{1}{\lambda_{m}}, 0\right),
$$

where $\eta$ is a constant determined to satisfy $\sum_{m=1}^{M} p_{m}=1$. This equation means that more power is allocated to a channel that has a larger eigenvalue $\left(p_{1} \geq \cdots \geq p_{M} \geq 0\right)$. Equations (8) to (11) are used in this subsection to calculate the CDFs of the capacities $C_{\mathrm{SDM}}$ and $C_{\mathrm{E}-\mathrm{SDM}}$ of MIMO channels when the normalized total TX power is $20 \mathrm{~dB}$.

The CDFs of the capacities of the measured $2 \times 2$ and $4 \times 4$ MIMO channels are shown in Figs. 12 and 13, respectively. In each figure, data for the $\mathrm{TX}-x / \mathrm{RX}-x$ and TX- $y / \mathrm{RX}-y$ orientations are shown in parts (a) and (b). All the $C_{\mathrm{SDM}}$ and $C_{\mathrm{E}-\mathrm{SDM}}$ in the LOS condition are higher than in the NLOS condition. It is clear from (9) and (10) that channel capacities $C_{\mathrm{SDM}}$ and $C_{\mathrm{E}-\mathrm{SDM}}$ are given by the eigenvalues, and by comparing the capacity distributions shown in Figs. 12 and 13 with the eigenvalue distributions shown in Figs. 10 and 11 we can see that the CDFs of capacities and eigenvalues display similar behavior. In short, it is clear that a MIMO case giving larger eigenvalues has a greater capacity than one giving smaller eigenvalues. Also, like the CDFs of eigenvalues, the CDFs of channel capacities in the LOS condition appear to depend on the LOS gain variation.

Comparing $C_{\mathrm{E}-\mathrm{SDM}}$ with $C_{\mathrm{SDM}}$, we can see that in both the LOS and NLOS conditions the $C_{\mathrm{E}-\mathrm{SDM}}$ is a little higher in the low cumulative frequency region. This improvement of the channel capacities is thought to be due to the optimum power coefficients that the WF theorem allocates to eigenchannels. Moreover, the improvement in the $4 \times 4$ MIMO system is greater than that in the $2 \times 2$ MIMO system. Investigating the improvement of $C_{\mathrm{E}-\mathrm{SDM}}$ from $C_{\mathrm{SDM}}$ at the $1 \%$ level, however, we cannot say that the effects of the WF theorem are clear for any of the MIMO cases. The range of improvement is about $0.3-0.4 \mathrm{bps} / \mathrm{Hz}$ in the $2 \times 2 \mathrm{MIMO}$ system and about $0.5-0.7 \mathrm{bps} / \mathrm{Hz}$ in the $4 \times 4$ MIMO system, respectively. Therefore, the abovementioned dependence of channel capacities on the LOS gain variation is almost the same in $C_{\mathrm{SDM}}$ and $C_{\mathrm{E}-\mathrm{SDM}}$.

The percentages of used substreams (eigenchannels) in $C_{\text {E-SDM }}$ at the normalized total TX power of $20 \mathrm{~dB}$ are shown in Fig. 14 for the measured $4 \times 4$ MIMO channels. Each pie chart presents the proportion of substreams to which the WF theorem given by (11) allocates positive power coefficients $\left(p_{m}>0\right)$. That is, it shows the relative usages of various numbers of the available eigenchannels. We can see that none of the MIMO systems utilize a single substream or two substreams, and that the $4 \times 4$ MIMO systems utilize three- or four-substream parallel transmission. The proportion of four-substream transmission is the largest and is greater in the LOS condition than in the NLOS condition. As demonstrated by the eigenvalue CDFs shown in Fig. 11, not only the maximum eigenvalues $\lambda_{1}$ but also $\lambda_{2}$, $\lambda_{3}$, and $\lambda_{4}$ tend to be larger in the LOS condition. Hence the probability that the WF theorem distributes the TX power even to the substream corresponding to the minimum eigenvalue $\lambda_{4}$ is higher in the LOS condition. In other words, the control by the WF theorem works in such a way that it utilizes more substreams. The results shown in (9) and (10) indicate that $C_{\mathrm{SDM}}$ and $C_{\mathrm{E}-\mathrm{SDM}}$ are similar when all four of the substreams are utilized. The measured data confirms that four-substream transmission increases the E-SDM channel capacity by $0-0.5 \mathrm{bps} / \mathrm{Hz}$ and that three-substream transmission increases it by $0.4-1.3 \mathrm{bps} / \mathrm{Hz}$ compared to the SDM channel capacity. Since the four-substream transmission that uses all of the eigenvalues from $\lambda_{1}$ to $\lambda_{4}$ is mainly used in the LOS condition, the performance of SDM and ESDM tends to be similar. Although we do not show the pie charts for the $2 \times 2$ MIMO system, the proportions of used substreams in $C_{\mathrm{E}-\mathrm{SDM}}$ indicate that in that system the twosubstream transmission accounts for over $95 \%$ in the LOS condition and over $90 \%$ of all transmission in the NLOS condition.

\subsection{BER Performance}

In the previous subsection, we evaluated the performance of E-SDM by using the channel capacity and compared it with the performance of the conventional SDM. The channel ca- 


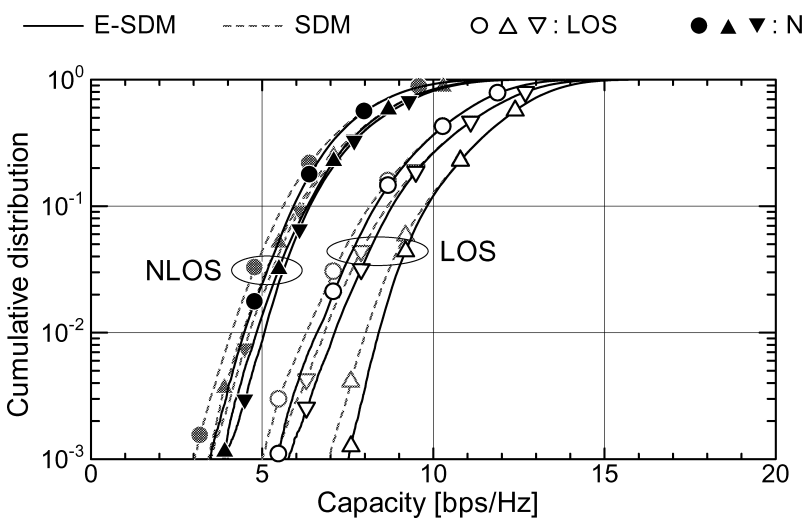

(a) TX- $x / \mathrm{RX}-x$.
O• : AS $=0.50 \lambda \quad \Delta \boldsymbol{\Delta}: \mathrm{AS}=0.75 \lambda \quad \nabla \boldsymbol{\nabla}: \mathrm{AS}=1.00 \lambda$

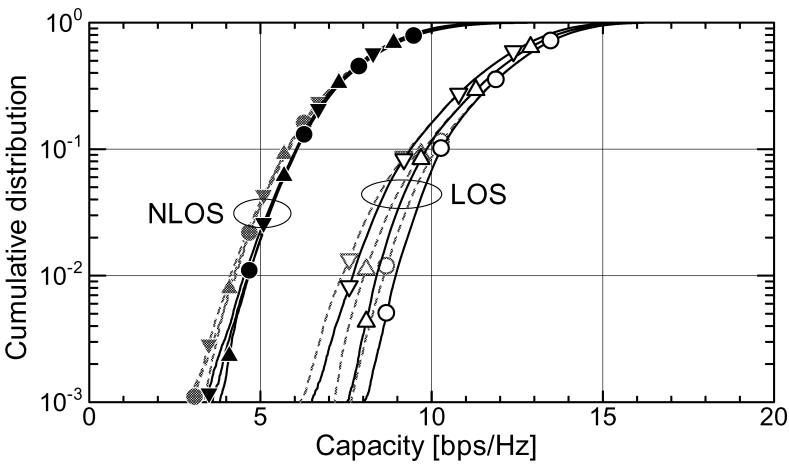

(b) TX- $y / \mathrm{RX}-y$.

Fig. 12 CDFs of channel capacities $C_{\mathrm{SDM}}$ and $C_{\mathrm{E}-\mathrm{SDM}}$ with a normalized total TX power of $20 \mathrm{~dB}$ for the measured $2 \times 2$ MIMO channels.

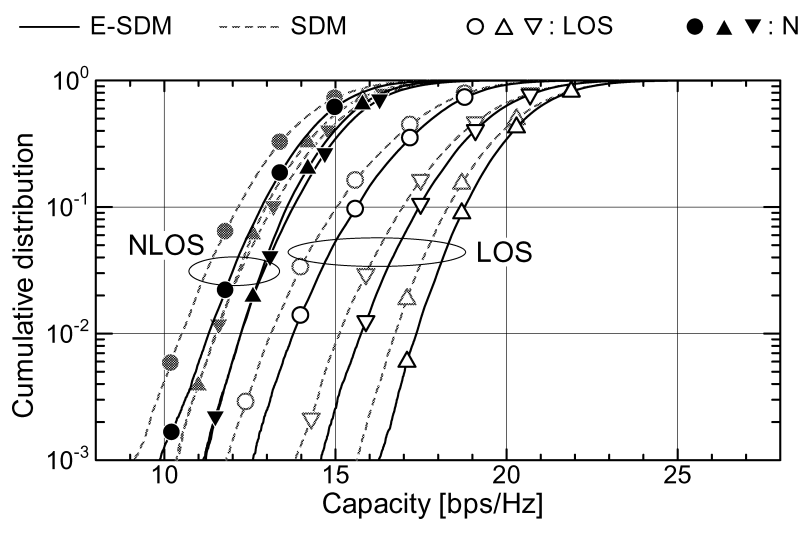

(a) TX- $x / \mathrm{RX}-x$. $\circ \bullet: A S=0.50 \lambda \quad \Delta \boldsymbol{\Delta}: A S=0.75 \lambda \quad \nabla \boldsymbol{\nabla}: A S=1.00 \lambda$

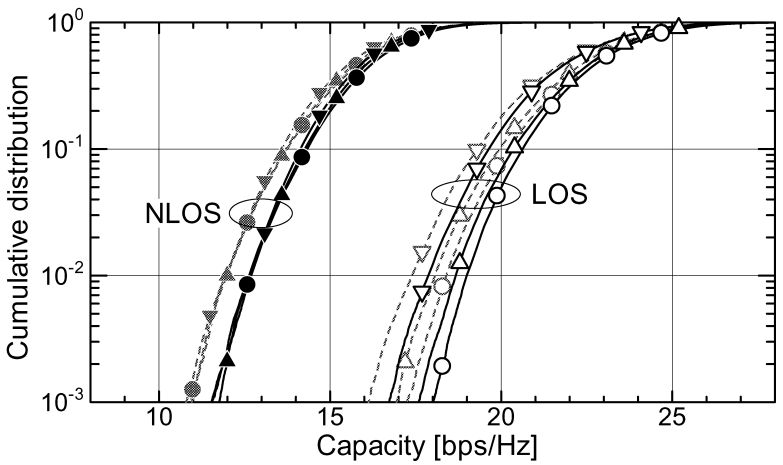

(b) $\mathrm{TX}-y / \mathrm{RX}-y$.

Fig. 13 CDFs of channel capacities $C_{\mathrm{SDM}}$ and $C_{\mathrm{E}-\mathrm{SDM}}$ with a normalized total TX power of $20 \mathrm{~dB}$ for the measured $4 \times 4$ MIMO channels.
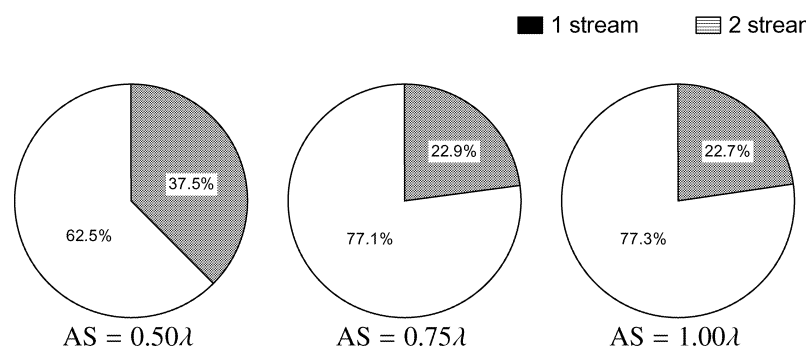

(a) TX- $x / \mathrm{RX}-x$, LOS.
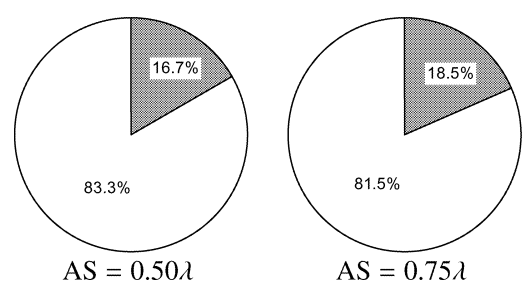

(c) TX- $y / \mathrm{RX}-y$, LOS.

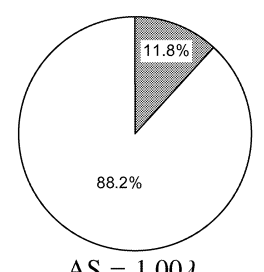

$\mathrm{AS}=1.00 \lambda$

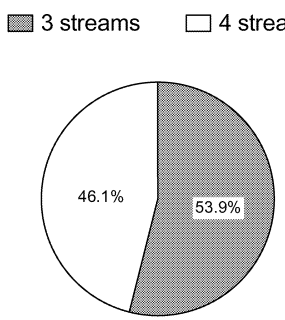

$\mathrm{AS}=0.50 \lambda$

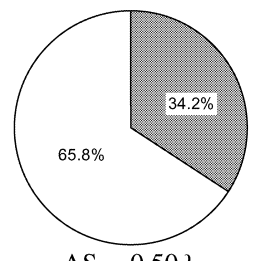

$\mathrm{AS}=0.50 \lambda$

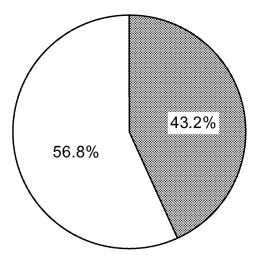

$\mathrm{AS}=0.75 \lambda$

(b) TX- $x / \mathrm{RX}-x$, NLOS

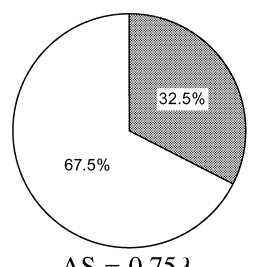

(d) TX-y/RX-y, NLOS.

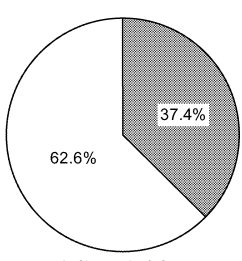

$\mathrm{AS}=1.00 \lambda$

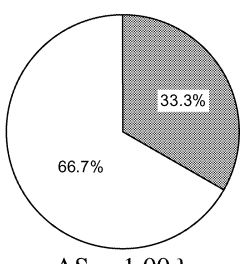

$\mathrm{AS}=1.00 \lambda$

Fig. 14 Percentages of substreams in E-SDM determined according to the WF theorem at the normalized total TX power of $20 \mathrm{~dB}$ for the measured $4 \times 4$ MIMO channels.

pacity given by (10) is a continuous quantity, and the number of used eigenchannels and their power allocation can be optimally determined by the WF theorem. The amount of information transmitted through each eigenchannel is a dis- 
Table 1 MIMO E-SDM and SDM simulation parameters.

\begin{tabular}{|c||c|c|}
\hline & E-SDM & SDM \\
\hline \hline Modulation & $\begin{array}{c}\text { QPSK, 16QAM, 64QAM, and 256QAM } \\
\text { (QPSK and 16QAM in the 2 } \times 2 \text { MIMO system) }\end{array}$ & QPSK \\
\hline Resource control & $\begin{array}{c}\text { Minimum BER criterion } \\
\text { based on Chernoff upper-bound }\end{array}$ & - \\
\hline RX signal processing & \multicolumn{2}{|c|}{ MRC } \\
\hline Data rates & $2 N_{T}$ bits/symbol \\
\hline Burst length & 128 symbols (no coding) \\
\hline Thermal noise & White Gaussian noise \\
\hline
\end{tabular}

crete quantity, however, and cannot actually be controlled on the basis of the WF theorem. From the implementational viewpoint, therefore, a concept of bit assignment and TX power allocation based on minimization of BER averaged over substreams has been proposed [20]. We examined the average BER performance of E-SDM transmission based on that resource control method by using computer simulations.

The simulation parameters are listed in Table 1. As stated previously, we had obtained 78,449 channel data for each MIMO configuration and averaged all the BERs. We conducted simulations for E-SDM and also did for SDM for comparison. In SDM we assumed that an independent QPSK-modulated uncoded substream was transmitted with equal power from each TX antenna. In other words, a total of $2 N_{T}$ bits/symbol were constantly transmitted (4 bits/symbol in the $2 \times 2 \mathrm{MIMO}$ and 8 bits/symbol in the $4 \times 4 \mathrm{MIMO}$ ). In the simulations for E-SDM the transmitter was also assumed to send data with a fixed rate of $2 N_{T}$ bits/symbol. Under this condition, in the $2 \times 2$ MIMO ESDM we selected modulations from QPSK and 16QAM and in the $4 \times 4$ MIMO E-SDM we selected modulations from QPSK, 16QAM, 64QAM, and 256QAM. Thus $2 \times 2$ MIMO E-SDM had the following two selection patterns:

- 16 QAM $\times 1\left(\lambda_{1}\right)$

- $\mathrm{QPSK} \times 2\left(\lambda_{1}, \lambda_{2}\right)$

And $4 \times 4$ MIMO E-SDM had the following five selection patterns:

- 256QAM $\times 1\left(\lambda_{1}\right)$

- 64QAM $\times 1\left(\lambda_{1}\right)+$ QPSK $\times 1\left(\lambda_{2}\right)$

- $16 \mathrm{QAM} \times 2\left(\lambda_{1}, \lambda_{2}\right)$

- $16 \mathrm{QAM} \times 1\left(\lambda_{1}\right)+\mathrm{QPSK} \times 2\left(\lambda_{2}, \lambda_{3}\right)$

- $\operatorname{QPSK} \times 4\left(\lambda_{1}, \lambda_{2}, \lambda_{3}, \lambda_{4}\right)$

From these patterns we selected the combination expected to give the minimum BER averaged over utilized substreams by using the Chernoff upper-bound. Then Lagrange multipliers determined the optimum TX power coefficients for the substreams [20]. Maximum likelihood detection (MLD) was used for RX signal processing in the SDM simulations because it is the method that gives the best performance [23]. The E-SDM simulations also assumed that the TX and RX sides have perfect CSI, whereas the SDM simulations assumed that only the RX side has perfect CSI.

The average BER performances of $2 \times 2$ and $4 \times$
4 MIMO systems are shown in Figs. 15 and 16, respectively. Looking at the E-SDM BER performance, we see - as we saw when comparing channel capacities (Figs. 12 and 13) that under the same TX power condition the LOS condition gives better performance than the NLOS condition does. The availability of conventional SDM in LOS environments has already been reported [11], [15], [16], [26] ${ }^{\dagger}$. E-SDM transmission never suffers degradation due to a high channel correlation, which is one of the issues in SDM (fading correlations for each MIMO configuration have been reported in Refs. [16], [26]). In LOS environments, eigenvalues become larger as the received power increases, and we have better performance in E-SDM. The better performance in the LOS condition is thought to be due to the direct wave.

In Fig. 17, we show percentages of bit and substream assignments in $4 \times 4$ MIMO E-SDM at the normalized total TX power of $20 \mathrm{~dB}$ in order to explain some phenomena raised below. The determination of these assignments was based on the minimum BER criterion used in this simulation. Unlike the proportion of used substreams based on the WF theorem shown in Fig. 14, when the minimum BER criterion is used, most of the transmission is two- or three-substream transmission and there is no four-substream transmission. Furthermore, while the WF theorem selects mostly four-substream transmission in the LOS condition, the minimum BER criterion selects fewer-substream transmission more frequently in the LOS condition than in the NLOS one. This means that when discrete digital information is transmitted by forming eigenbeams, the BER performance obtained when sending much information through fewer eigenchannels with larger eigenvalues is better than that obtained when using many eigenchannels. This phenomenon is considered to be the difference between an ideal case given by the information theory and an actual case with limited finite modulations.

The differences in channel capacities between SDM and E-SDM are not large as described in the previous subsection. The BER performance obtained with E-SDM, in

'In Refs. [15], [16], [26] and this paper, the authors fairly compare the MIMO-SDM performance in the LOS and NLOS condition under the same TX power constraint, and report that LOS channels provide better performance due to higher received power. Note that, however, some NLOS performances outperform LOS ones under the same SNR condition, where fading correlations determine major performance of MIMO-SDM. 


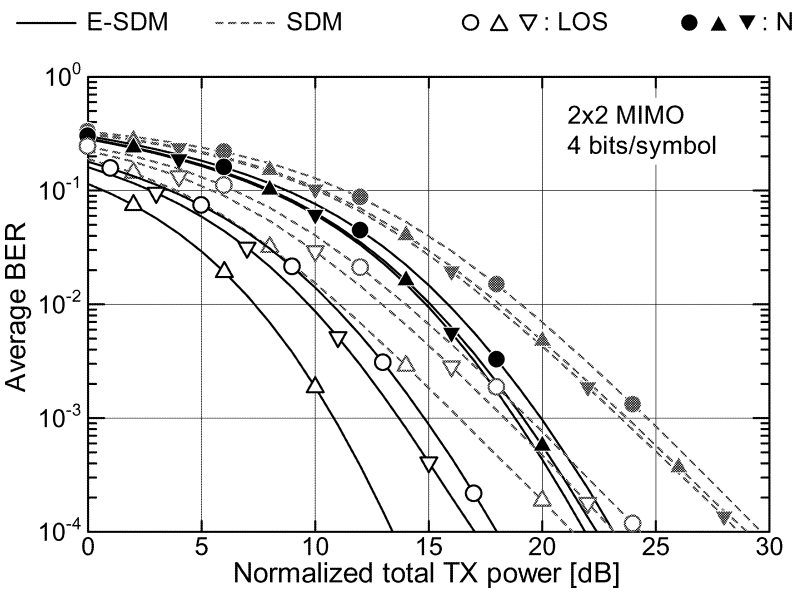

(a) $\mathrm{TX}-x / \mathrm{RX}-x$.

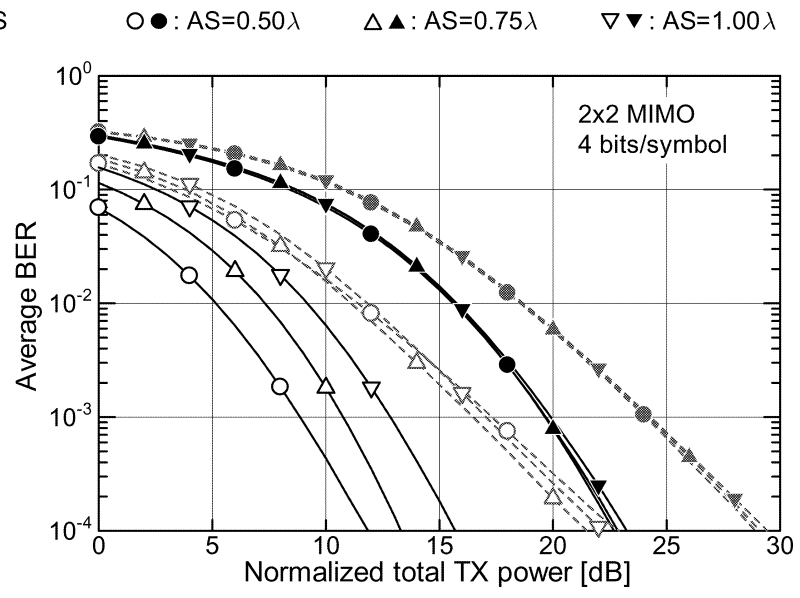

(b) $\mathrm{TX}-y / \mathrm{RX}-y$.

Fig. 15 Average BER performance of SDM and E-SDM for the measured $2 \times 2$ MIMO channels.

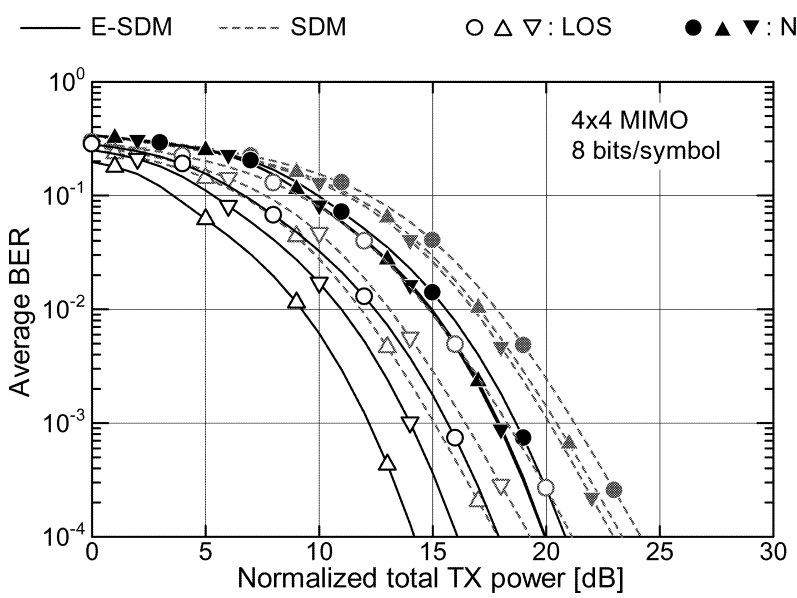

(a) $\mathrm{TX}-x / \mathrm{RX}-x$.
O• : $\mathrm{AS}=0.50 \lambda \quad \triangle \boldsymbol{\Delta}: \mathrm{AS}=0.75 \lambda$

$\nabla \nabla: A S=1.00 \lambda$

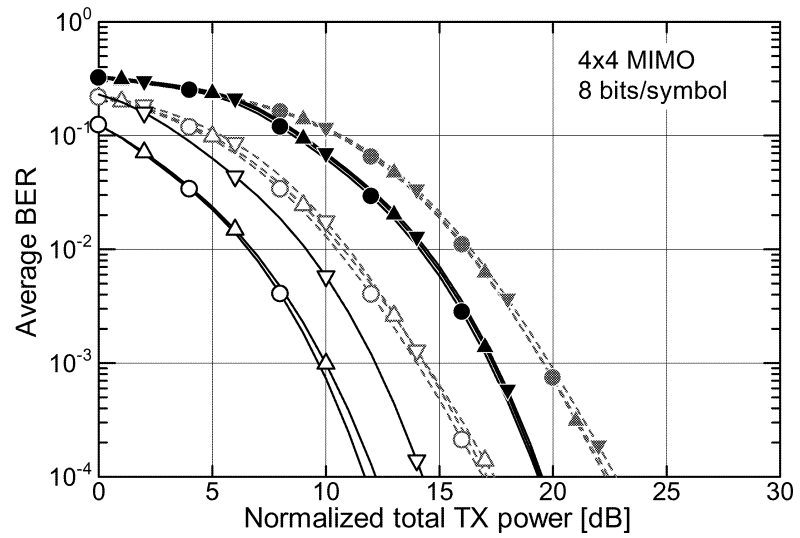

(b) TX-y/RX- $y$.

Fig. 16 Average BER performance of SDM and E-SDM for the measured $4 \times 4$ MIMO channels.

256QAM (1 stream) 首 64QAM + QPSK (2 streams) $\quad$ 血 16QAM×2 (2 streams) $\square$ 16QAM + QPSK×2 (3 streams) $\square$ QPSK×4 (4 streams)

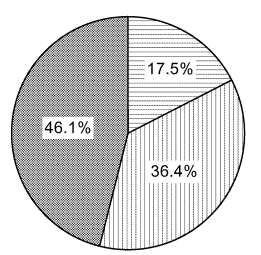

$\mathrm{AS}=0.50 \lambda$

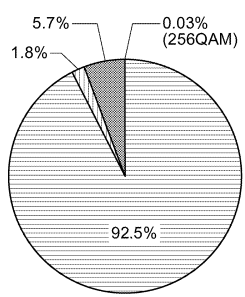

$\mathrm{AS}=0.50 \lambda$

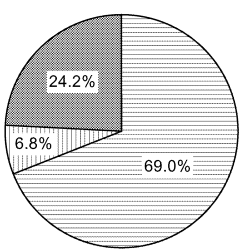

$\mathrm{AS}=0.75 \lambda$ (a) $\mathrm{TX}-x / \operatorname{RX}-x$, LOS.

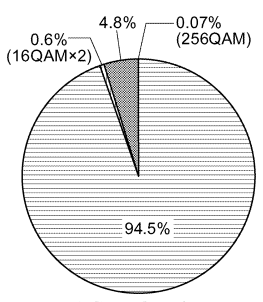

$\mathrm{AS}=0.75 \lambda$ (c) TX- $y /$ RX- $y$, LOS.

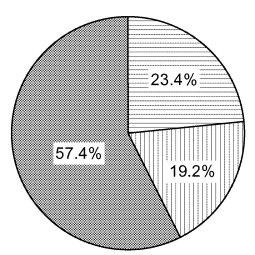

$\mathrm{AS}=1.00 \lambda$

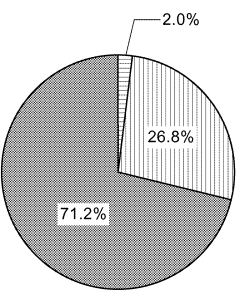

$\mathrm{AS}=1.00 \lambda$

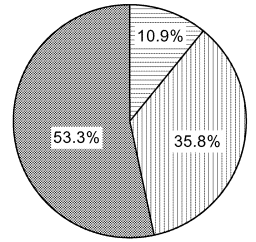

$\mathrm{AS}=0.50 \lambda$

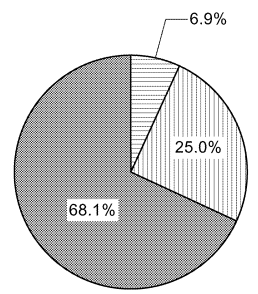

$\mathrm{AS}=0.50 \lambda$

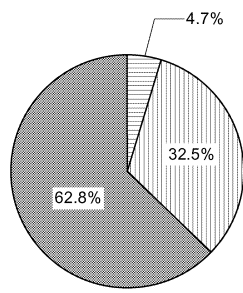

$\mathrm{AS}=0.75 \lambda$

(b) $\mathrm{TX}-x / \mathrm{RX}-x$, NLOS.

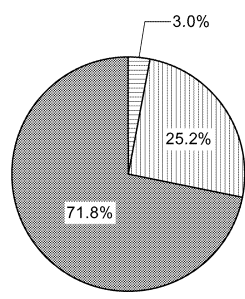

$\mathrm{AS}=0.75 \lambda$

(d) $\mathrm{TX}-y / \mathrm{RX}-y$, NLOS.

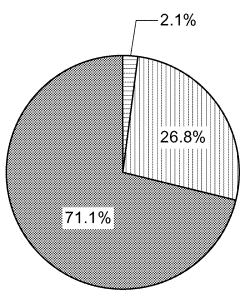

$\mathrm{AS}=1.00 \lambda$

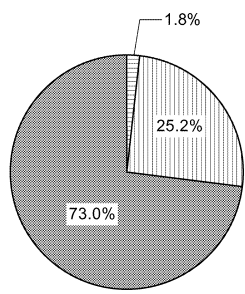

$\mathrm{AS}=1.00 \lambda$

Fig. 17 Percentages of substreams in E-SDM determined according to the minimum BER criterion at the normalized total TX power of $20 \mathrm{~dB}$ for the measured $4 \times 4$ MIMO channels. 
contrast, clearly excels in all of the MIMO configurations. This is considered to be due to fewer-substream transmission mentioned above. As stated in Sect. 4.2, overall minimum eigenvalues have noticeably lower values than the others. Hence, unlike the WF theorem, the minimum BER criterion appears to judge minimum eigenvalues unavailable. In addition, Figs. 10 and 11 show that eigenchannels provide spatial diversity gain except the one with the minimum eigenvalue as described also in Sect. 4.2. Under a constant bit rate, advantages of more diversity gain obtained by choosing fewer-substream transmission seem to greatly outweigh the degradation caused by multilevel modulation schemes such as 16QAM and 64QAM.

The LOS gain variation appears to cause differences also in E-SDM BER performance and to result in a variety of trends in resource allocation, especially for $4 \times 4$ MIMO systems. For example, when AS $=0.50 \lambda$ or $0.75 \lambda$ in the $\mathrm{TX}-y / \mathrm{RX}-y$ orientation in the LOS condition, predominant transmission is the two-substream transmission (in which 64QAM modulation is allocated to the first eigenchannel with the maximum eigenvalue $\lambda_{1}$ and QPSK modulation is allocated to the second eigenchannel with $\lambda_{2}$ ). Moreover, it is only in these cases that we find one-substream transmission in which 256QAM is allocated to the first eigenchannel. The first eigenchannel thus plays an important role in these cases. We can see in Fig. 11(c) that the eigenvalues $\lambda_{1}$ for these two cases are remarkably large. As stated in Sects. 4.1 and 4.2, the large values of $\lambda_{1}$ are due to higher gain in the $90^{\circ}$ direction. Since the higher gain was caused by the mutual coupling in antennas, we can say that the BER performance of E-SDM tends to be affected by the mutual coupling effect. For this reason, the E-SDM performance in the TX- $y / \mathrm{RX}-y$ array orientation in the LOS condition consequentially improves as the AS decreases.

Although as in the previous subsection we do not present figures showing the percentages of substreams for the $2 \times 2$ MIMO system, we confirmed that about $80 \%$ of the transmission in the NLOS condition is one-substream transmission utilizing only 16QAM. The percentage of onesubstream transmission in the LOS condition also differs depending on the array configuration. In the TX- $x / \mathrm{RX}-x$ orientation it is $87.6 \%$ for $\mathrm{AS}=0.50 \lambda, 93.6 \%$ for $\mathrm{AS}=0.75 \lambda$, and $85.8 \%$ for AS $=1.00 \lambda$, while in the TX- $y / \mathrm{RX}-y$ orientation it is $99.2 \%$ for $\mathrm{AS}=0.50 \lambda, 92.2 \%$ for $\mathrm{AS}=0.75 \lambda$, and $74.2 \%$ for $\mathrm{AS}=1.00 \lambda$. As in the $4 \times 4 \mathrm{MIMO}$ system, the results in the $2 \times 2$ MIMO system, that are obtained when the minimum BER criterion is used, differ from those obtained when the WF theorem is used.

\section{Conclusions}

We have evaluated the performance of MIMO E-SDM in the $5.2 \mathrm{GHz}$ frequency band by using channel data measured in indoor LOS and NLOS environments. CDFs of channel capacities and average bit error rates were examined with array element patterns and CDFs of eigenvalues, and were compared with those of conventional SDM.
From the results, we found that the LOS condition, in which there is the direct wave, gives better E-SDM performance. And although channel capacities did not indicate clear benefits of E-SDM, we obtained excellent BER performance by employing E-SDM compared with conventional SDM. We also found that the maximum channel capacity criterion (WF theorem) and the minimum BER criterion have different characteristics with regard to TX resource allocation. Moreover, in the LOS condition the BER performance especially in E-SDM strongly depends on the MIMO configuration.

\section{Acknowledgement}

This work was supported in part by a Research Fellowship for Young Scientists from the Japan Society for the Promotion of Science.

\section{References}

[1] D. Gesbert, M. Shafi, D.S. Shiu, P. Smith, and A. Naguib, "From theory to practice: An overview of MIMO space-time coded wireless systems," IEEE J. Sel. Areas Commun., vol.21, no.2, pp.281-302, April 2003.

[2] A.J. Paulraj, D.A. Gore, R.U. Nabar, and H. Bölcskei, "An overview of MIMO communications-A key to gigabit wireless," Proc. IEEE, vol.92, no.2, pp.198-218, Feb. 2004.

[3] I.E. Telatar, "Capacity of multi-antenna Gaussian channels," Euro. Trans. Telecommun., vol.1, no.6, pp.585-595, Nov./Dec. 1999.

[4] A.F. Molisch, M. Steinbauer, M. Toeltsch, E. Bonek, and R.S. Thomä, "Capacity of MIMO systems based on measured wireless channels," IEEE J. Sel. Areas Commun., vol.20, no.3, pp.561569, April 2002.

[5] J.P. Kermoal, L. Schumacher, K.I. Pedersen, P.E. Mogensen, and F. Frederiksen, "A stochastic MIMO radio channel model with experimental validation," IEEE J. Sel. Areas Commun., vol.20, no.6, pp.1211-1226, Aug. 2002.

[6] H. Özcelik, M. Herdin, H. Hofstetter, and E. Bonek, "Capacity of different MIMO systems based on indoor measurements at 5.2 GHz," Proc. EPMCC 2003, pp.463-466, April 2003.

[7] P. Kyritsi, D.C. Cox, R.A. Valenzuela, and P.W. Wolniansky, "Correlation analysis based on MIMO channel measurements in an indoor environment," IEEE J. Sel. Areas Commun., vol.21, no.5, pp.713720, June 2003

[8] T. Mitsui, M. Otani, H.Y.E. Chua, K. Sakaguchi, and K. Araki, "Indoor MIMO channel measurements for evaluation of effectiveness of array antenna configurations," Proc. IEEE VTC2003-Fall, vol.1, pp.84-88, Oct. 2003.

[9] K. Yu, M. Bengtsson, B. Ottersten, D. McNamara, P. Karlsson, and M. Beach, "Modeling of wide-band MIMO radio channels based on NLoS indoor measurements," IEEE Trans. Veh. Technol., vol.53, no.3, pp.655-665, May 2004.

[10] K. Nishimori, N. Tachikawa, Y. Takatori, R. Kudo, and K. Tsunekawa, "Frequency correlation characteristics due to antenna configurations in broadband MIMO transmission," IEICE Trans. Commun., vol.E88-B, no.6, pp.2438-2445, June 2005.

[11] K. Sakaguchi, H.Y.E. Chua, and K. Araki, "MIMO channel capacity in an indoor line-of-sight (LOS) environment," IEICE Trans. Commun., vol.E88-B, no.7, pp.3010-3019, July 2005.

[12] G.D. Golden, G.J. Foschini, R.A. Valenzuela, and P.W. Wolniansky, "Detection algorithm and initial laboratory results using V-BLAST space-time communication architecture," Electron. Lett., vol.35, no.1, pp.14-16, Jan. 1999. 
[13] G.J. Foschini, G.D. Golden, R.A. Valenzuela, and P.W. Wolniansky, "Simplified processing for high spectral efficiency wireless communication employing multi-element arrays," IEEE J. Sel. Areas Commun., vol.17, no.11, pp.1841-1852, Nov. 1999.

[14] G.J. Foschini, D. Chizhik, M.J. Gans, C. Papadias, and R.A. Valenzuela, "Analysis and performance of some basic space-time architectures," IEEE J. Sel. Areas Commun., vol.21, no.3, pp.303320, April 2003.

[15] H. Nishimoto, Y. Ogawa, T. Nishimura, and T. Ohgane, "Performance of MIMO-SDM in indoor line-of-sight environments based on $5.2 \mathrm{GHz}$ measurements," Proc. ISAP'04, 2A4-4, pp.345-348, Aug. 2004.

[16] H. Nishimoto, Y. Ogawa, T. Nishimura, and T. Ohgane, "Availability of MIMO spatial multiplexing in line-of-sight channels," Proc. IEEE VTS APWCS 2005, pp.40-44, Aug. 2005.

[17] K. Ban, M. Katayama, T. Yamazato, and A. Ogawa, "Joint optimization of transmitter/receiver with multiple transmit/receive antennas in band-limited channels," IEICE Trans. Commun., vol.E83-B, no.8, pp.1697-1704, Aug. 2000.

[18] H. Sampath, P. Stoica, and A. Paulraj, "Generalized linear precoder and decoder design for MIMO channels using the weighted MMSE criterion,” IEEE Trans. Commun., vol.49, no.12, pp.2198-2206, Dec. 2001

[19] A. Scaglione, P. Stoica, S. Barbossa, G.B. Giannakis, and H. Sampath, "Optimal designs for space-time linear precoders and decoders," IEEE Trans. Signal Process., vol.50, no.5, pp.1051-1064, May 2002.

[20] K. Miyashita, T. Nishimura, T. Ohgane, Y. Ogawa, Y. Takatori, and K. Cho, "High data-rate transmission with eigenbeam-space division multiplexing (E-SDM) in a MIMO channel," Proc. IEEE VTC2002Fall, vol.3, pp.1302-1306, Sept. 2002.

[21] Y. Karasawa, "MIMO propagation channel modeling," IEICE Trans Commun., vol.E88-B, no.5, pp.1829-1842, May 2005.

[22] W. Weichselberger, M. Herdin, H. Özcelik, and E. Bonek, "A stochastic MIMO channel model with joint correlation of both link ends," IEEE Trans. Wireless Commun., vol.5, no.1, pp.90-100, Jan. 2006.

[23] T. Ohgane, T. Nishimura, and Y. Ogawa, "Applications of space division multiplexing and those performance in a MIMO channel," IEICE Trans. Commun., vol.E88-B, no.5, pp.1843-1851, May 2005.

[24] J.W. Wallace and M.A. Jensen, "Mutual coupling in MIMO wireless systems: A rigorous network theory analysis," IEEE Trans. Wireless Commun., vol.3, no.4, pp.1317-1325, July 2004.

[25] J.G. Proakis, Digital Communications, 3rd ed., McGraw-Hill, New York, 1995

[26] Y. Ogawa, H. Nishimoto, T. Nishimura, and T. Ohgane, "Performance of MIMO spatial multiplexing in indoor line-of-sight environments," Proc. IEEE VTC2005-Fall, vol.4, pp.2398-2402, Sept. 2005.

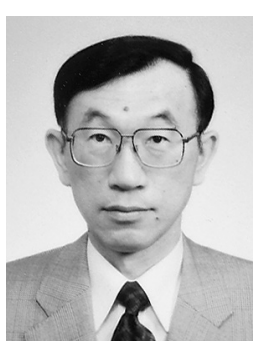

Yasutaka Ogawa received the B.E., M.E. and Ph.D. degrees from Hokkaido University, Sapporo, Japan, in 1973, 1975, and 1978, respectively. Since 1979, he has been with Hokkaido University, where he is currently a Professor of the Graduate School of Information Science and Technology. During 1992-1993, he was with ElectroScience Laboratory, the Ohio State University, U.S.A., as a Visiting Scholar, on leave from Hokkaido University. His interests are in adaptive antennas, mobile communications, super-resolution techniques, and MIMO systems. Dr. Ogawa is a senior member of the IEEE.

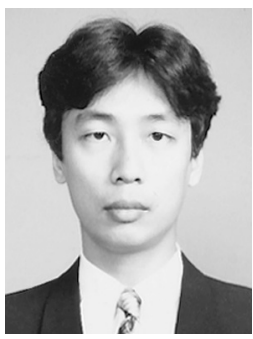

Toshihiko Nishimura received the B.S and M.S. degrees in physics and Ph.D. degree in electronics engineering from Hokkaido University, Sapporo, Japan, in 1992, 1994, and 1997, respectively. In 1998, he joined the Graduate School of Engineering (reorganized to Graduate School of Information Science and Technology at present) at Hokkaido University, where he is currently a Research Associate of Electronics and Information Engineering Division. His current research interests are in the MIMO system using smart antenna. Dr. Nishimura is a member of the IEEE and the Physical Society of Japan.

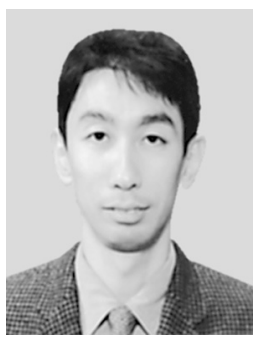

Takeo Ohgane received the B.E., M.E., and $\mathrm{Ph} . \mathrm{D}$. degrees in electronics engineering from Hokkaido University, Sapporo, Japan, in 1984, 1986, and 1994, respectively. From 1986 to 1992, he was with Communications Research Laboratory, Ministry of Posts and Telecommunications. From 1992 to 1995 , he was on assignment at ATR Optical and Radio Communications Research Laboratory. Since 1995, he has been with Hokkaido University, where he is an Associate Professor. During 2005-2006, he was at Centre for Communications Research, University of Bristol, U.K., as a Visiting Fellow. His interests are in MIMO signal processing for wireless communications. He received the IEEE AP-S Tokyo Chapter Young Engineer Award in 1993. Dr. Ohgane is a member of the IEEE.

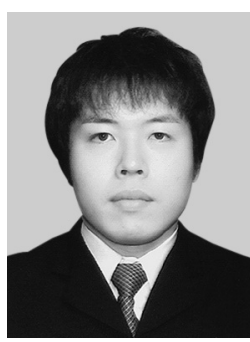

Hiroshi Nishimoto received the B.E. and M.E. degrees from Hokkaido University, Sapporo, Japan, in 2003 and 2005, respectively. He is currently working towards the Ph.D. degree at the Graduate School of Information Science and Technology, Hokkaido University. His research interests are in MIMO propagation measurement and MIMO communication systems. He has been a Research Fellow of the Japan Society for the Promotion of Science since 2005. $\mathrm{He}$ is a student member of the IEEE. 